\title{
Journal review
}

\section{Follow-up of CREST trial: no difference in restenosis rates between stenting and surgery \\ doi: 10.1016/S0019-4832(12)60068-6}

It should sound like music to interventionalists. The new results from carotid revascularisation endarterectomy versus stenting trial (CREST) show that after 2 years of follow-up, restenosis rates are equal whether the patient underwent stenting or surgery. The data was presented at New Orleans recently during the International Stroke conference by Dr. Brajesh K. Lal. The rates of restenosis/occlusion were 6\% and $6.3 \%$ in the stenting and surgery groups respectively (difference not statistically significant). The CREST trial is one of the recent and well-discussed trials in the area of carotid stenting and surgery that enrolled 2500 patients from 117 US and Canadian centres. The composite end-point was a combination of any stroke, myocardial infarction (MI), or death during the peri-procedural period and ipsilateral stroke during follow-up. Stenting was associated with a $7.2 \%$ event rate versus $6.8 \%$ with surgery, again non-significant difference. However, the stroke was higher with stenting while MI was higher with endarterectomy. The present analysis showed that during the follow-up by Doppler ultrasound, similar rates of restenosis (>70\% diameter reduction)/occlusion were demonstrated between the stenting and surgery arms. The risk factors for restenosis in this analysis showed that women and patients with diabetes mellitus or dyslipidemia had double the risk.

2. The PROFI study (prevention of cerebral embolisation by proximal balloon occlusion compared to filter protection during carotid artery stenting). A prospective randomised trial.

Bijuklic K, Wandler A, Hazizi F, Schofer J.

J Am Coll Cardiol Jan 25, 2012.

doi: 10.1016/S0019-4832(12)60069-8

Background: Randomised trials comparing filter-protected carotid artery stenting (CAS) with carotid endarterectomy revealed a higher peri-procedural stroke rate after CAS. Proximal balloon occlusion may be more effective in preventing cerebral embolisation during CAS than filters.

Methods: Patients undergoing CAS with cerebral embolic protection for internal carotid artery stenosis were randomly assigned to proximal balloon occlusion or filter protection.
The primary endpoint was the incidence of new cerebral ischaemic lesions assessed by diffusion-weighted magnetic resonance imaging (MRI). Secondary endpoints were the number and volume of new ischaemic lesions and major adverse cardiovascular and cerebral events (MACCE).

Results: Sixty-two consecutive patients (mean age: 71.7 years, $76.4 \%$ male) were randomised. Compared with filter protection $(n=31)$, proximal balloon occlusion $(n=31)$ resulted in a significant reduction in the incidence of new cerebral ischaemic lesions ( $45.2 \%$ vs $87.1 \%, P=0.001)$. The number (median [range]: 2 [0-13] vs 0 [0-4], $P=0.0001)$ and the volume $\left(0.47[0-2.4] \mathrm{cm}^{3}\right.$ vs $\left.0[0-0.84] \mathrm{cm}^{3}, P=0.0001\right)$ of new cerebral ischaemic lesions were significantly reduced by proximal balloon occlusion. Lesions in the contralateral hemisphere were found in $29 \%$ and $6.5 \%$ of patients (filter vs balloon occlusion, respectively, $P=0.047$ ). The 30 -day MACCE rate was $3.2 \%$ and $0 \%$ for filter versus balloon occlusion, respectively ( $P=$ non-significant).

Conclusion: In this randomised trial of patients undergoing CAS, proximal balloon occlusion as compared with filter protection significantly reduced the embolic load to the brain.

\section{Perspective}

Further strength for the use of the proximal protection device has been provided by the PROFI study. The distal protection devices have certain inherent disadvantages:

1. As the device crosses the lesion, in the beginning, there is a small risk of embolisation before deployment.

2. The distal protection devices (DPD) may not capture very tiny emboli.

3. If the DPD is not abutting the vessel wall, some emboli may still pass.

4. When there is large embolisation, the filter may clog which may lead to embolisation during retrieval.

A proximal balloon occlusion device like MO.MA does not have most of these disadvantages. However, there are issues like bulky design, higher cost and of course, the device is less operator-friendly. Also, it is contraindicated in cases of contralateral carotid occlusion.

The authors of PROFI study have demonstrated that the risk of embolisation is considerably reduced by using the MO.MA device. They randomised 62 patients to proximal versus distal protection (Emboshield, Abbott) devices and by using 